\title{
Affinage et qualité du Gruyère de Comté \\ IV. Etude de la protéolyse
}

\author{
J.C. COLLIN, J.L. BERDAGUÉ*, Mai DOGNIN-BERGERET** \\ et R. GRAPPIN \\ avec la collaboration technique de Marie-Pierre MUTTONI \\ et Odile ROLET-REPECAUD \\ INRA, Station expérimentale laitière, 39800 Poligny, France \\ * Institut Technique du Gruyère, B.P. no 1010, 01009 Bourg-en-Bresse, France \\ ** Faculté des Sciences, La Bouloie, 25030 Besançon Cedex, France
}

\section{Résumé}

Cette étude sur l'affinage du Gruyère de Comté, portant sur quatre fromageries, quatre caves d'affinage et six fabrications réparties sur deux saisons, permet de mesurer l'influence de ces facteurs sur la qualité du produit fini. Au total 96 fromages sont analysés à quatre stades successifs de leur affinage.

La répétabilité du dosage quantitatif des caséines par électrophorèse est mesurée à partir de 700 résultats. Elle varie linéairement en fonction de la valeur des résultats, de $2,13 \%$ à $5,64 \%$.

Trois semaines après la fabrication du fromage, $78,8 \%$ des caséines $\alpha \mathrm{S} 1$ et $84,2 \%$ des caséines $\beta$ ne sont pas hydrolysées. Ces proportions sont encore de $23 \%$ pour les $\alpha$ S1 et de $39,8 \%$ pour les $\beta$ en fin d'affinage.

L'étude des fractions caséine montre qu'il est possible de définir deux ensembles de variables relativement indépendants : les caséines $\beta, \beta$ deg. ( 2 bandes électrophorétiques correspondant à des produits de dégradation de la caséine $\beta$ ), $\gamma 2$, et $\gamma 3$ forment le premier ensemble tandis que les $\alpha$ S1 et $\alpha$ S1I forment le second. La fraction $\gamma 1$ apparaît indépendante des $\gamma 2$ et $\gamma 3$. La variation des $\alpha \mathrm{S} 1$ est très liée à la température de la cave d'affinage. L'influence du facteur fromagerie (composition du lait, nature des ferments lactiques, technologie...) est importante sur les fractions NS (fraction azotée soluble dans l'eau), $\beta, \beta$ deg., $\gamma 2, \gamma 3$ et $\alpha$ S1.

Le facteur saison influe de façon significative sur les produits de dégradation de la caséine $\beta$. Des mécanismes différents de la protéolyse selon la saison et en fonction de la température de la cave d'affinage sont proposés. L'évolution comparée du taux de plasmine et des proportions des $\gamma$ et $\beta$ deg. dans les laits au cours des six mois étudiés est montrée à deux stades différents de l'affinage des fromages.

Mots clés: Gruyère de Comté - Affinage - Protéolyse - Electrophorèse - Caséine Plasmine - Saison - Température.

Ce programme a reçu le soutien financier de la Direction des Industries Agricoles et Alimentaires du Ministère de l'A griculture (contrat DIAA $\mathrm{n}^{\circ}$ 81/27) et de l'Association Nationale pour la Recherche et la Technologie (Bourse CIFRE de J.L. Berdagué, convention $\mathbf{n}^{\circ}$ 92/82). 


\section{Summary}

\section{Ripening of Gruyère de Comté cheese. IV. Study of proteolysis}

The ripening of Gruyère de Comté cheese was studied using four dairies capable of making four identical cheeses from the same vat of milk. Each of these 4 cheeses was ripened in one of four different ripening rooms. Six trials were conducted during six different months on two seasons. As a whole 96 cheeses were analysed at four successive stages of their maturation. Determinations of plasmine contents of milks were performed as well as quantitative evolutions of cheese protein fractions by polyacrylamide-agarose gel electrophoresis.

The repeatability of the electrophoretic determinations was tested from 700 measurements. It varied linearely according to the casein levels between 2.13 and 5.64

After 3 weeks of ripening, $78.8 \%$ of $\alpha \mathrm{S} 1$ caseins and $84.2 \%$ of $\beta$ caseins remained unhydrolysed, and at the end of ripening, $23.0 \%$ of $\alpha \mathrm{S} 1$ caseins and $39.8 \%$ of $\beta$ caseins were still unhydrolysed.

The study showed that casein fractions could be divided into two independant groups: a first group containing the $\beta-$, deg. $\beta-(2$ unidentified electrophorectic fractions released from $\beta$-casein), $\gamma 2$ - and $\gamma 3$-casein fractions and a second group containing the $\alpha \mathrm{S} 1 \mathrm{I}$ and $\alpha \mathrm{S} 1$ fractions.

The $\gamma 1$ fraction appeared to be independant of the $\gamma 2$ and $\gamma 3$-caseins. The amount of $\alpha \mathrm{S} 1$ was closely related to the temperature of the ripening room. The influence of dairy factors (milk composition, starters, technology...) was important on the NS (water soluble peptide fraction), $\beta-$, deg. $\beta-, \gamma 2-, \gamma 3-$ and $\alpha$ S1-fractions. Seasons significantly affected the products of degradation of $\beta$-casein. Different mechanisms of hydrolysis seemed to exist depending on the season and the temperature of the ripening room. The evolution of plasmin levels in milk were compared with the proportions of $\gamma$ - and deg. $\beta$-fractions at two stages of the ripening of cheeses.

Key words : Comté Gruyère cheese - Ripening - Proteolysis - Electrophoresis - Casein Plasmin - Season - Temperature.

\section{Introduction}

La dégradation enzymatique des caséines, pendant la période d'affinage d'un fromage, joue un rôle déterminant en libérant des composés qui contribuent à donner au fromage sa texture et son arôme caractéristiques. L'affinage traditionnel du Gruyère de Comté, qui est un fromage à pâte cuite pressée, s'effectue dans la majorité des cas à des températures comprises entre 10 et $20{ }^{\circ} \mathrm{C}$ et pendant des durées de 3 à 6 mois. La protéolyse des fromages est un phénomène complexe à cause de la diversité des protéases mises en jeu. Ces protéases ont des origines différentes; elles proviennent du lait (principalement la plasmine), des enzymes coagulantes (la chymosine et la pepsine bovine) et des microorganismes. C'est ainsi que les microorganismes se développant soit à l'intérieur du fromage soit sur sa croûte naturelle (ou morge) peuvent participer à la dégradation des protéines fromagères. 
L'action de nombreuses protéases sur les caséines in vitro et au cours de l'affinage des fromages a beaucoup été étudiée au cours des dix dernières années (GrapPin et al., 1985). La vitesse de dégradation des caséines au cours de l'affinage dépend largement des conditions technologiques utilisées lors de la fabrication du fromage. Gripon et al. (1975) montrent, par exemple, que dans un fromage de type Saint Paulin "aseptique " la présure a pratiquement entièrement dégradé la caséine $\alpha \mathrm{S} 1$ en fin d'affinage. Dans les fromages à pâte cuite pressée comme le Comté, la présure est fortement inactivée (GARNOT et Molé́, 1987) et nous avons peu d'informations sur la nature et l'importance de la protéolyse dans ce type de fromage. Au cours de ce travail, qui s'inscrit dans un programme plus vaste sur le rôle de l'affinage sur la qualité du Comté (GrapPIN et al., 1987 ; Berdagué, 1986), nous étudions la nature et la vitesse de la protéolyse durant l'affinage du Gruyère de Comté ainsi que l'incidence de la fromagerie, de la saison de fabrication et des conditions d'affinage sur les proportions des différentes caséines.

\section{Matériel et méthodes}

\section{A. Matériel}

Plan d'expérience : l'étude porte sur quatre fromageries (F1, F2, F3 et F4) dont les fromages sont affinés habituellement par quatre affineurs (A1, A2, A3 et A4) ayant des techniques et des conditions d'affinage différentes (voir l'article de Grappin et al., 1987). Chaque fromagerie possède une cuve multiple à quatre fromages et, lors de chaque fabrication, chacun des 4 fromages, considérés comme ayant un comportement et des caractéristiques identiques, est affiné chez un affineur différent.

Les fabrications sont répétées six fois, réparties sur deux périodes ou saisons (février, mars, avril et août, septembre, octobre). Au total 96 fromages sont analysés ( 4 fromageries $\times 4$ affineurs $\times 2$ saisons $\times 3$ mois).

Les fromages au cours de leur maturation sont analysés à quatre stades : stade $\mathrm{F}$ (fromagerie : 20 heures après la fabrication du fromage), stade $\mathrm{A}$ (avant affinage : 3 semaines après la fabrication et début de l'affinage industriel), stade $\mathrm{P}$ (pendant l'affinage : 1 mois et demi après la fabrication) et stade C (coupe du fromage : 4 à 10 mois après la fabrication selon le type d'affinage). Au stade coupe, chez un affineur donné, les 4 fromages provenant des 4 fromageries sont coupés simultanément. Les échantillons de fromage sont râpés pour obtenir un échantillon homogène et sont conservés à $-25^{\circ} \mathrm{C}$.

\section{B. Méthodes}

\section{Fractions azotées}

Les techniques analytiques employées sont les suivantes : l'azote total (N) selon la méthode Kjeldahl, l'azote soluble dans l'eau (NS), qui représente la majeure partie des fractions azotées non visibles en électrophorèse, d'après la 
méthode de Kuchroo et Fox (1982) et l'azote soluble dans l'acide phosphotungstique (NPT), c'est-à-dire les petits peptides et les acides aminés, selon la technique décrite par Gripon et al. (1975).

\section{Analyse électrophorétique des fractions caséines}

On ajoute $20 \mathrm{ml}$ de tampon citrate de sodium $0,1 \mathrm{M}(\mathrm{pH} 7,0)$ à $0,5 \mathrm{~g}$ de fromage. Un broyage de 3 minutes est réalisé à l'aide d'un broyeur ultrarapide (Ultra-Turrax). Le mélange est ensuite acidifié à $\mathrm{pH} 4,4$ à l'aide d'une solution d'acide chlorhydrique $1 \mathrm{~N}$. Après quelques minutes d'agitation, les protéines précipitées sont récupérées par centrifugation $(10000 \mathrm{~g})$. Le culot est dissout dans $5 \mathrm{ml}$ d'urée $9 \mathrm{M}$ contenant $1 \%$ de $\beta$-mercapto-éthanol.

Les électrophorèses sont effectuées dans un gel d'acrylamide-bisacrylamide-agarose $(5-0,5-0,8 \%)$ en tampon Tris-glycine $(0,075 \mathrm{M}-0,06 \mathrm{M} ; \mathrm{pH}$ 8,6 ) contenant $4,5 \mathrm{M}$ d'urée et $0,2 \%$ de $\beta$ - mercapto-éthanol (Trieu-Cuot et GRIPON, 1983). L'échantillon en urée $9 \mathrm{M}$ est mélangé avec un volume égal d'agarose $4 \%$. Dix microlitres de ce mélange maintenu à $40{ }^{\circ} \mathrm{C}$ sont introduits dans un réservoir préalablement creusé dans le gel. L'électrophorèse a lieu à $+10^{\circ} \mathrm{C}$ pendant $2 \mathrm{~h} 30$ sous une tension de $25 \mathrm{~V}$ par $\mathrm{cm}$.

Les protéines sont précipitées puis colorées par le bleu de Coomassie R 250 (URIEL, 1966) et le gel est décoloré dans le mélange éthanol-acide acétique-glycérol-eau (200/50/25/725; V/V/V/V).

Une analyse quantitative des plaques est effectuée après déshydratation du gel sur un densitomètre enregistreur (Vernon). La surface de chaque pic est mesurée et exprimée en pourcentage de la surface totale de l'ensemble des pics.

\section{Dosage de la plasmine}

La plasmine est dosée selon la technique de Richardson et PeArce (1981). Les résultats sont exprimés en unités de substrat AMC consommées par $\mathrm{ml}$ de lait (1 unité $\mathrm{AMC}=10^{-9}$ Mole d'amino-méthyl-coumarine par minute et par $\mathrm{ml}$ de lait).

\section{Méthodes statistiques}

Les analyses statistiques utilisées sont décrites dans l'article de Grappin et al. (1987). Elles ont été réalisées d'une part à l'aide de la programmathèque Amance (BACHACOU et al., 1981) et d'autre part à partir des logiciels élaborés par Microstat ITCF (1984).

\section{Résultats et discussion}

\section{A. Identification des principales protéines}

Le résultat de l'électrophorèse d'un échantillon de lait et d'un échantillon du fromage fabriqué avec ce lait, à quatre stades successifs de son affinage, est 
montré dans la figure 1 . Les quatre principales caséines du lait ( $\alpha$ S1, $\alpha$ S2, $\beta$ et $\kappa$ ) sont très facilement identifiables. Dans les échantillons de fromage, la bande migrant au-delà de la caséine $\alpha \mathrm{S} 1$ provient de la dégradation des $\alpha \mathrm{S} 1$; elle est appelée $\alpha$ S1I (Fox et Guiney, 1973; Mulvihill et Fox, 1980). Cette protéine correspond à la partie C terminale (24-199) de la caséine $\alpha \mathrm{S} 1$ (Creamer et Richardson, 1974).

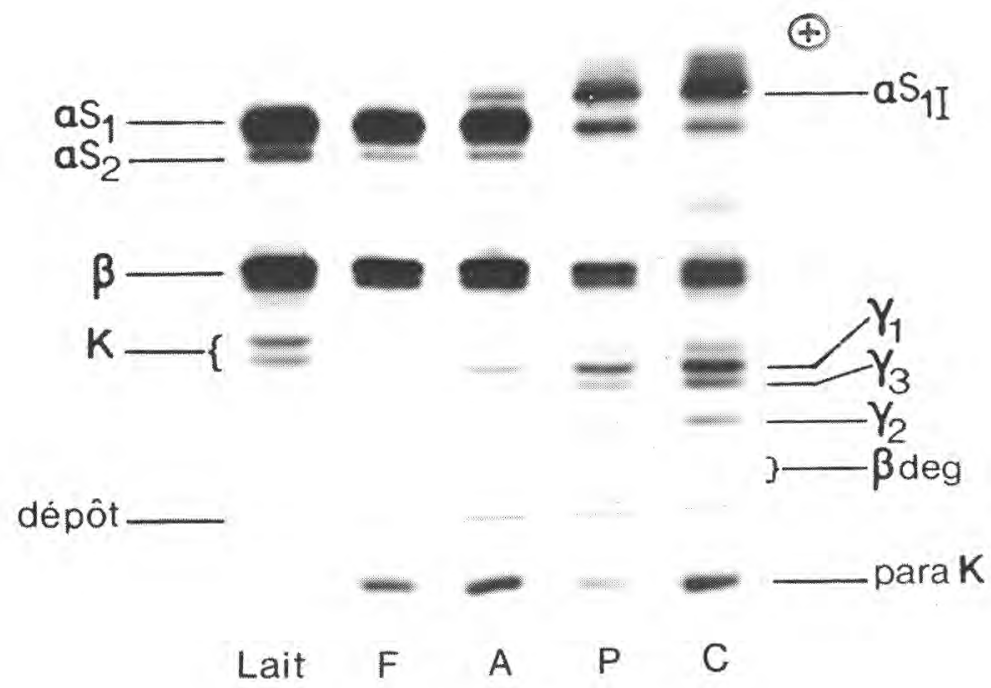

Fig. 1

Analyse électrophorétique d'un échantillon de lait et d'un échantillon de fromage à quatre stades successifs de son affinage.

Electrophoretic analysis of milk and cheese samples at four successive stages of the ripening process.

Les trois caséines $\gamma$ sont très peu visibles dans le lait mais facilement identifiables dans le fromage. Elles correspondent à la partie $\mathrm{C}$ terminale de la caséine $\beta$ : $\gamma 1$ (29-209), $\gamma 2$ (106-209) et $\gamma 3$ (108-208) (Creamer, 1974).

La première double bande visible après le dépôt provient probablement de la dégradation de la caséine $\beta$ selon Trieu-CuOT et Gripon (1983); nous l'avons appelée $\beta$ deg. ( $\beta$ dégradée). La para-caséine $\kappa$, bien que très visible n'a pas été incorporée dans notre étude car son apparition après coloration est apparue très irrégulière.

\section{B. Linéarité et reproductibilité des résultats}

L'électrophorèse en gel de polyacrylamide agarose est une technique sensible qui a beaucoup été utilisée pour des déterminations qualitatives et 
beaucoup plus rarement pour des déterminations quantitatives. Nous avons donc voulu déterminer la proportionnalité entre la réponse du spectrophotomètre et la quantité de protéines déposées pour chaque caséine étudiée.

La figure 2 représente les réponses du spectrophotomètre obtenues avec des quantités croissantes de caséine $\beta$. Les linéarités obtenues avec toutes les caséines ont été très satisfaisantes. Dans notre étude, nous avons voulu en priorité mettre en évidence les évolutions des quantités de caséines au cours de l'affinage et les différences entre fromageries ou caves d'affinage, c'est pourquoi nous avons négligé les différences de capacité de fixation du colorant par les diverses caséines. D'ailleurs, les caractéristiques de fixation du colorant pour toutes les protéines étudiées ne sont pas connues.

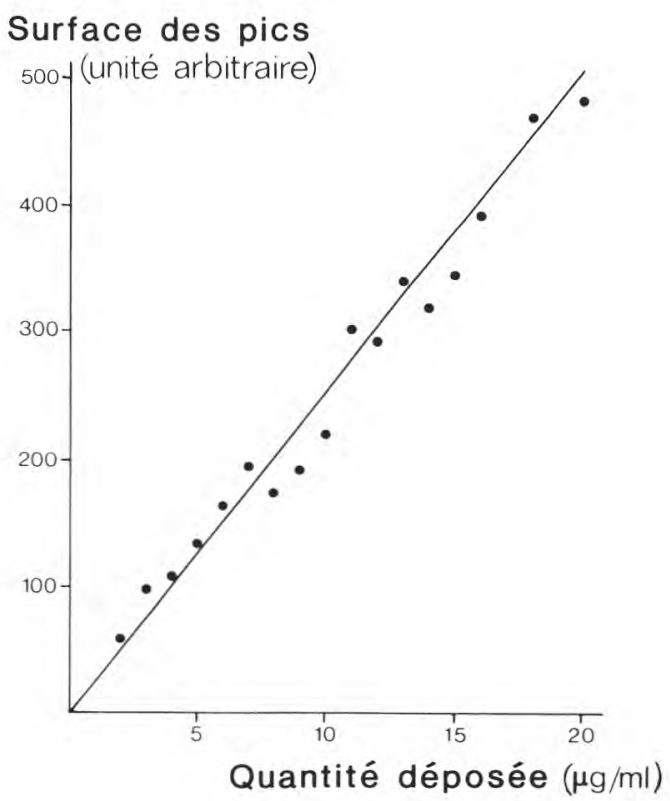

Fig. 2

Relation entre la réponse du spectrophotomètre et la quantité de caséine $\beta$ dans l'échantillon analysé.

Relationship between the spectrophotometric measurement and the amount of $\beta$ casein in the analysed sample.

Le calcul de répétabilité de cette méthode électrophorétique a retenu toute notre attention car il s'agissait de mettre en évidence des différences de teneurs en caséines entre fromageries ou entre caves d'affinage qui pouvaient être très faibles.

Le tableau 1 , calculé à partir de 700 résultats obtenus en double sur des fromages aux stades $\mathrm{P}$ et $\mathrm{C}$, indique l'écart-type de répétabilité, la répétabilité 
(exprimée par l'écart maximal obtenu entre deux doubles) et le coefficient de variation, en fonction de la moyenne des valeurs obtenues. Ce tableau montre que pour une faible valeur de $3 \%$, le résultat sera compris entre $1,54 \%$ et $4,46 \%$ et pour une forte valeur de $25 \%$, le résultat sera compris entre $21,15 \%$ et $28,85 \%$ et ceci dans $95 \%$ des cas.

\section{TABLEAU 1}

Répétabilité de la méthode électrophorétique

Repeatability of the electrophoretic method

\begin{tabular}{|c|c|c|c|c|c|c|}
\hline \multicolumn{2}{|c|}{$\begin{array}{c}\text { Protéines } \\
\text { (en \% de la surface } \\
\text { totale des pics) }\end{array}$} & $0-5$ & $5-10$ & $10-15$ & $15-20$ & sup. 20 \\
\hline \multicolumn{2}{|r|}{ Moyenne $(\bar{x})$} & 3,10 & 7,40 & 12,2 & 17,8 & 25,9 \\
\hline \multirow{3}{*}{ 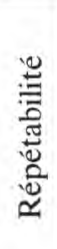 } & Ecart-type ( $\sigma r)$ & 0,753 & 0,934 & 1,21 & 1,32 & 1,99 \\
\hline & $\mathrm{r}=2,83 \cdot \sigma \mathrm{r}$ & 2,13 & 2,64 & 3,42 & 3,75 & 5,64 \\
\hline & C.V. $=\frac{\sigma r}{\bar{x}} \times 100$ & 24,3 & 12,6 & 9,9 & 7,4 & 7,7 \\
\hline
\end{tabular}

Il existe une excellente relation linéaire entre l'écart-type de répétabilité et la moyenne des valeurs obtenues. Ainsi la répétabilité de la méthode varie linéairement avec la valeur mesurée $(\mathrm{x})$ selon l'équation $\sigma \mathrm{r}=0,055 \mathrm{x}+0,55$ avec un coefficient de corrélation de 0,98 . L'utilisation de spectrophotomètre plus discriminant à rayon laser par exemple devrait augmenter la précision de la lecture et donc la précision de cette méthode d'analyse.

\section{Variabilité et composition moyenne des fromages}

Les résultats obtenus en électrophorèse ainsi que les résultats de dosage des fractions azotées sont regroupés dans les tableaux 2 et 3.

D'une manière générale, on constate logiquement une augmentation continue des fractions NS, NPT, $\alpha$ S1I, $\beta$ deg., $\gamma 1, \gamma 2$ et $\gamma 3$ au cours de l'affinage. A l'inverse, les fractions $\alpha$ S1 et $\beta$ diminuent.

Les teneurs en NS et en NPT, qui sont des indicateurs généraux de la protéolyse, sont multipliées respectivement par 6,5 et 10,2 entre la fabrication et la fin d'affinage pour les 96 fromages.

Les taux de NS et NPT sont de l'ordre de 1,49 et 0,61 dans les fromages prêts à être commercialisés, ce qui correspond à $21,6 \%$ et $8,9 \%$ de l'azote total (tabl. 2). Les protéines détectées en électrophorèse représentent des produits de poids moléculaire important (supérieur à 10000 daltons) et seront 
TABLEAU 2

Résultats moyens, obtenus sur les 96 fromages, du dosage de l'azote soluble dans l'eau

(NS) et dans l'acide phosphotungstique (NPT) aux 4 stades d'affinage, exprimés en pourcent de l'extrait sec (colonne 1) et en pourcent de l'azote total (colonne 2)

Average results, from 96 cheese samples, of the determination of water soluble nitrogen

(NS) and of phosphotungstic acid nitrogen (NPT) at 4 ripening stages, expressed

as percent of dry matter (column 1) and as percent of total nitrogen (column 2)

\begin{tabular}{|c|c|c|c|c|c|c|c|}
\hline & \multirow{2}{*}{$\begin{array}{l}\frac{\pi}{\pi} \\
\frac{\pi}{\infty}\end{array}$} & \multicolumn{2}{|c|}{ Moyenne } & \multirow{2}{*}{\multicolumn{2}{|c|}{ Valeurs extrêmes }} & \multirow{2}{*}{ Ecart-type } & \multirow{2}{*}{ C.V. } \\
\hline & & (1) & (2) & & & & \\
\hline \multirow{4}{*}{ NS } & $\mathrm{F}$ & 0,23 & 3,30 & 0,14 & 0,30 & 0,04 & 18,91 \\
\hline & A & 0,50 & 7,25 & 0,33 & 0,67 & 0,10 & 20,38 \\
\hline & $\mathrm{P}$ & 1,07 & 15,53 & 0,59 & 1,60 & 0,20 & 18,45 \\
\hline & $\mathrm{C}$ & 1,49 & 21,65 & 1,27 & 1,93 & 0,13 & 8,80 \\
\hline \multirow{4}{*}{ NPT } & $\mathrm{F}$ & 0,06 & 0,86 & 0,03 & 0,08 & 0,01 & 19,90 \\
\hline & A & 0,13 & 1,88 & 0,07 & 0,19 & 0,03 & 25,68 \\
\hline & $\mathrm{P}$ & 0,34 & 4,95 & 0,14 & 0,74 & 0,11 & 32,10 \\
\hline & $\mathrm{C}$ & 0,61 & 8,87 & 0,40 & 0,99 & 0,14 & 21,60 \\
\hline
\end{tabular}

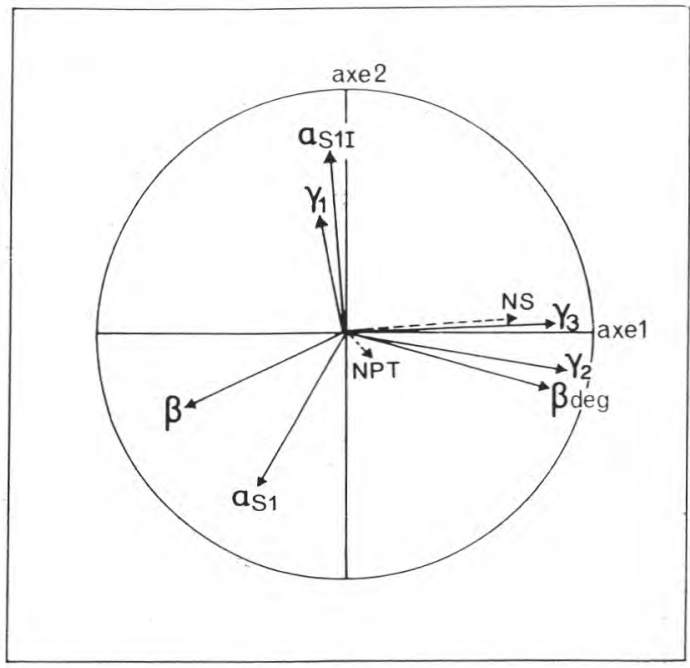

Fig. 3

Cercle des corrélations obtenu à partir des résultats du dosage des différentes fractions caséine effectué en fin d'affinage.

Circle of correlations obtained from results of electrophoretic analysis of different casein fractions at the end of ripening. 
plus ou moins dégradées au cours de l'affinage. Ainsi, à un instant déterminé, les valeurs mesurées représentent pour une protéine intermédiaire $(\alpha$ S1I, $\gamma 1$, $\gamma 2, \gamma 3$ et $\beta$ deg.) un équilibre entre la quantité formée et la quantité hydrolysée. Les taux mesurés entre la fabrication et la fin d'affinage augmentent d'un facteur 9,4 pour les $\beta$ deg., de 5,3 pour les $\gamma 1$, de 3,8 pour les $\gamma 2$ et de 2,8 pour les $\gamma 3$ (tabl. 3 ).

\section{TABLEAU 3}

Résultats moyens, obtenus sur les 96 fromages, du dosage électrophorétique des principales fractions caséine aux quatre stades d'affinage, exprimés en pour cent des caséines totales

Average results, obtained with 96 cheese samples, of electrophoretic analysis of the principal casein fractions at four ripening stages, expressed as percent of whole casein

\begin{tabular}{|c|c|c|c|c|c|c|}
\hline & Stades & Moyenne & \multicolumn{2}{|c|}{ Valeurs extrêmes } & Ecart-type & C.V. \\
\hline$\alpha \mathrm{S} 1$ & $\begin{array}{l}\mathrm{F} \\
\mathrm{A} \\
\mathrm{P} \\
\mathrm{C}\end{array}$ & $\begin{array}{l}48,81 \\
39,80 \\
21,94 \\
13,78\end{array}$ & $\begin{array}{r}42,46 \\
27,46 \\
14,25 \\
7,54\end{array}$ & $\begin{array}{l}59,90 \\
55,95 \\
37,39 \\
21,79\end{array}$ & $\begin{array}{l}3,69 \\
6,97 \\
5,14 \\
2,77\end{array}$ & $\begin{array}{r}7,57 \\
17,51 \\
23,45 \\
20,11\end{array}$ \\
\hline$\alpha \mathrm{S} 11$ & $\begin{array}{l}\mathrm{F} \\
\mathrm{A} \\
\mathrm{P} \\
\mathrm{C}\end{array}$ & $\begin{array}{r}4,71 \\
12,50 \\
25,57 \\
28,68\end{array}$ & $\begin{array}{r}0,00 \\
0,85 \\
13,65 \\
22,61\end{array}$ & $\begin{array}{l}12,38 \\
21,86 \\
34,13 \\
36,38\end{array}$ & $\begin{array}{l}3,26 \\
5,16 \\
3,81 \\
2,72\end{array}$ & $\begin{array}{r}69,03 \\
41,32 \\
14,93 \\
9,49\end{array}$ \\
\hline$\beta$ & $\begin{array}{l}\mathrm{F} \\
\mathrm{A} \\
\mathrm{P} \\
\mathrm{C}\end{array}$ & $\begin{array}{l}38,39 \\
33,49 \\
24,83 \\
18,71\end{array}$ & $\begin{array}{l}31,78 \\
25,78 \\
17,30 \\
12,26\end{array}$ & $\begin{array}{l}45,90 \\
41,52 \\
33,31 \\
24,73\end{array}$ & $\begin{array}{l}2,87 \\
3,26 \\
3,51 \\
2,54\end{array}$ & $\begin{array}{r}7,47 \\
9,75 \\
14,11 \\
13,78\end{array}$ \\
\hline$\beta$ deg. & $\begin{array}{l}\mathrm{F} \\
\mathrm{A} \\
\mathrm{P} \\
\mathrm{C}\end{array}$ & $\begin{array}{l}0,28 \\
0,17 \\
1,92 \\
2,64\end{array}$ & $\begin{array}{l}0,00 \\
0,00 \\
0,00 \\
0,58\end{array}$ & $\begin{array}{l}3,77 \\
3,49 \\
4,99 \\
5,40\end{array}$ & $\begin{array}{l}0,79 \\
0,52 \\
1,04 \\
1,07\end{array}$ & $\begin{array}{c}282,74 \\
312,0 \\
54,24 \\
40,33\end{array}$ \\
\hline$\gamma 1$ & $\begin{array}{l}\mathrm{F} \\
\mathrm{A} \\
\mathrm{P} \\
\mathrm{C}\end{array}$ & $\begin{array}{l}1,80 \\
3,74 \\
8,26 \\
9,49\end{array}$ & $\begin{array}{l}0,00 \\
0,57 \\
4,75 \\
7,19\end{array}$ & $\begin{array}{r}3,46 \\
6,75 \\
10,40 \\
13,39\end{array}$ & $\begin{array}{l}0,93 \\
0,36 \\
1,24 \\
1,16\end{array}$ & $\begin{array}{r}51,85 \\
135,91 \\
15,08 \\
12,26\end{array}$ \\
\hline$\gamma 2$ & $\begin{array}{l}\text { F } \\
\text { A } \\
\text { P } \\
\text { C }\end{array}$ & $\begin{array}{l}1,71 \\
2,77 \\
4,51 \\
6,54\end{array}$ & $\begin{array}{l}0,21 \\
0,28 \\
0,89 \\
2,40\end{array}$ & $\begin{array}{r}3,40 \\
6,70 \\
7,94 \\
10,06\end{array}$ & $\begin{array}{l}0,79 \\
0,55 \\
1,73 \\
1,68\end{array}$ & $\begin{array}{r}45,96 \\
153,91 \\
38,42 \\
25,64\end{array}$ \\
\hline$\gamma 3$ & $\begin{array}{l}\mathrm{F} \\
\mathrm{A} \\
\mathrm{P} \\
\mathrm{C}\end{array}$ & $\begin{array}{l}1,21 \\
2,25 \\
5,50 \\
7,41\end{array}$ & $\begin{array}{l}0,09 \\
0,28 \\
2,08 \\
3,94\end{array}$ & $\begin{array}{l}2,41 \\
4,29 \\
8,25 \\
9,99\end{array}$ & $\begin{array}{l}0,60 \\
0,43 \\
1,49 \\
1,23\end{array}$ & $\begin{array}{l}50,03 \\
98,26 \\
27,07 \\
16,56\end{array}$ \\
\hline
\end{tabular}


Trois semaines après la fabrication du fromage, lorsque celui-ci entre en cave chaude chez l'affineur, seulement $21,2 \%$ de la caséine $\alpha$ S1 et $15,8 \%$ de la caséine $\beta$ sont hydrolysés. En fin d'affinage, à la coupe du fromage, ces proportions ne sont que de $77 \%$ pour la caséine $\alpha \mathrm{S} 1$ et $60,2 \%$ pour les caséines $\beta$.

\section{Relations entre les variables (taux azotés et taux protéiques)}

L'analyse en composantes principales des différentes fractions caséine mesurées au stade " coupe » du fromage a permis d'obtenir une vue synthétique des phénomènes protéolytiques impliquant ces fractions. Les fractions azotées NS et NPT ont été ajoutées au calcul comme variables supplémentaires.

L'étude du cercle de corrélations (fig. 3) obtenu dans le plan principal 1-2 (qui contribue à $62 \%$ de la variance totale) montre qu'il est possible de définir pour les fractions caséine, deux ensembles de variables relativement indépendantes. Les caséines $\beta, \beta$ deg., $\gamma 2$ et $\gamma 3$ sont fortement corrélées avec l'axe 1 et constituent le premier ensemble de variables, tandis que les fractions $\alpha$ S1 et $\alpha$ S1I sont bien représentées selon l'axe 2 et forment le second ensemble de variables.

La fraction $\gamma$, indépendante de l'ensemble des autres fractions $\gamma$, est surtout corrélée avec l'axe 3 et dans une moindre mesure avec l'axe 2 (tabl. 4).

Les fractions azote soluble (NS) apparaissent très corrélées à l'axe 1 et semblent se comporter de manière semblable aux fractions $\gamma 3, \gamma 2$ et $\beta$ deg. La fraction NPT est très mal représentée dans le plan étudié et n'est faiblement corrélée qu'avec des axes lointains indépendants des fractions caséine.

En définitive, l'étude du taux des caséines au stade coupe reflète les étroites corrélations ( $\mathrm{p}<0,001$ ) existant entre $\gamma 2, \gamma 3$ et $\beta$ deg., ainsi que les corrélations négatives plus faibles mais significatives $(p<0,001)$ entre $\beta$ et les fractions $\gamma_{2}(\mathrm{r}=-0,424), \quad \gamma 3(\mathrm{r}=-0,345)$ et $\beta$ deg. $\quad(\mathrm{r}=-0,338)$ (tabl. 4). De même une corrélation négative $(\mathrm{p}<0,001)$ est observée entre les fractions $\alpha$ S1 et $\alpha$ S1I ( $\mathrm{r}=-0,349)$. La fraction $\gamma 1$ n'est pas corrélée aux autres fractions caséine, excepté avec $\beta$ deg. $(r=-0,357, p<0,001)$.

\section{E. Influence des facteurs affinage, fromagerie et saison de fabrication sur les taux protéiques}

L'influence des facteurs affinage (A), fromagerie (F), saison (S) est estimée par une analyse de variance des fractions azotées et des fractions caséine. Le tableau 5 indique les résultats obtenus aux stades $P$ et $C$ de l'affinage du fromage.

\section{Facteur affinage}

Les différences obtenues entre les stades $\mathrm{P}$ et $\mathrm{C}$ avec le facteur affinage s'expliquent essentiellement par la différence de température d'affinage entre A1 et les 3 autres affineurs. Cette différence de l'importance du taux de 


\section{TABLEAU 4}

Coefficients de corrélations entre teneurs en fractions caséine au stade coupe et coefficients de corrélations entre teneurs en fractions caséine et les axes principaux de l'analyse en composantes principales (fig. 3)

Correlation coefficients between contents in casein fractions at the end of ripening, and correlation coefficients between casein fractions and the principal axis from the principal components analysis (fig. 3)

Corrélations

\begin{tabular}{l|r|r|r|r|r|r|r}
\hline & \multicolumn{1}{c|}{$\beta$ deg } & \multicolumn{1}{c|}{$\gamma 1$} & $\gamma 2$ & $\gamma 3$ & $\beta$ & $\alpha \mathrm{S} 1$ & $\alpha \mathrm{S} 1 \mathrm{I}$ \\
\cline { 2 - 7 }$\beta$ deg & 1,000 & & & & & & \\
$\gamma 1$ & $-0,357$ & 1,000 & & & & & \\
$\gamma 2$ & 0,798 & $-0,055$ & 1,000 & & & & \\
$\gamma 3$ & 0,620 & 0,143 & 0,876 & 1,000 & & & \\
$\beta$ & $-0,338$ & $-0,094$ & $-0,424$ & $-0,345$ & 1,000 & & \\
$\alpha$ S1 & $-0,334$ & $-0,074$ & $-0,260$ & $-0,247$ & 0,156 & 1,000 & \\
$\alpha$ S1I & $-0,086$ & 0,120 & 0,193 & $-0,026$ & $-0,138$ & $-0,349$ & 1,000 \\
\hline
\end{tabular}

Etude des variables

$1^{\text {re }}$ colonne : corrélations entre les variables et les axes principaux

$2^{\mathrm{e}}$ colonne : corrélation au carré.

\begin{tabular}{l|rr|rr|rr}
\hline \multirow{2}{*}{ Variables } & \multicolumn{6}{|c}{ Composantes principales } \\
\cline { 2 - 7 } & \multicolumn{2}{|c|}{ Axe 1 } & \multicolumn{2}{c}{ Axe 2 } & \multicolumn{2}{c}{ Axe 3} \\
\hline BD & 0,8695 & 0,7561 & $-0,2250$ & 0,0506 & 0,2859 & 0,0817 \\
$\gamma 1$ & $-0,0826$ & 0,0068 & 0,5769 & 0,3328 & $-0,7613$ & 0,5796 \\
$\gamma 2$ & 0,9493 & 0,9012 & $-0,1621$ & 0,0263 & $-0,1450$ & 0,0210 \\
$\gamma 3$ & 0,8682 & 0,7538 & 0,0333 & 0,0011 & $-0,2789$ & 0,0778 \\
$\beta$ & $-0,5506$ & 0,3032 & $-0,2705$ & 0,0732 & 0,1691 & 0,0228 \\
$\alpha$ S1 & $-0,4305$ & 0,1854 & $-0,5707$ & 0,3257 & $-0,4044$ & 0,1636 \\
$\alpha$ S1I & $-0,0332$ & 0,0011 & 0,7976 & 0,6362 & 0,3814 & 0,1455 \\
\hline
\end{tabular}

\begin{tabular}{l|rr|rr|rr}
\hline $\begin{array}{c}\text { Variables } \\
\text { supplémentaires }\end{array}$ & \multicolumn{2}{|c|}{ Axe 1 } & \multicolumn{2}{c}{ Axe 2 } & \multicolumn{2}{c}{ Axe 3 } \\
\hline NS & 0,7408 & 0,5488 & 0,0741 & 0,0055 & 0,0752 & 0,0057 \\
NPT & 0,1228 & 0,0151 & $-0,1038$ & 0,0108 & 0,2944 & 0,0867 \\
\hline
\end{tabular}


protéolyse aux premiers stades d'affinage est compensée au stade coupe par un allongement de la durée d'affinage pour l'affineur A1 (fig. 4).

Au stade coupe, seule la proportion de caséine $\alpha$ S1 est influencée de façon hautement significative par le facteur affinage. La courbe 5 montre les variations du pourcentage moyen des caséines $\alpha$ S1 selon les caves d'affinage au stade coupe en fonction de la température moyenne de ces caves d'affinage. Les trois caves A1, A2 et A3 affinent les fromages selon un seul palier de température alors que la cave 4 utilise 2 paliers. Le point A4 sur la courbe de la figure 5 représente la moyenne générale de la cave ; le point A'4 correspond au premier palier de température (environ deux tiers de la durée totale de l'affinage). Tout semble se passer comme si l'augmentation de la température du deuxième palier n'avait pas d'effet sur la protéolyse de la caséine $\alpha$ S1.

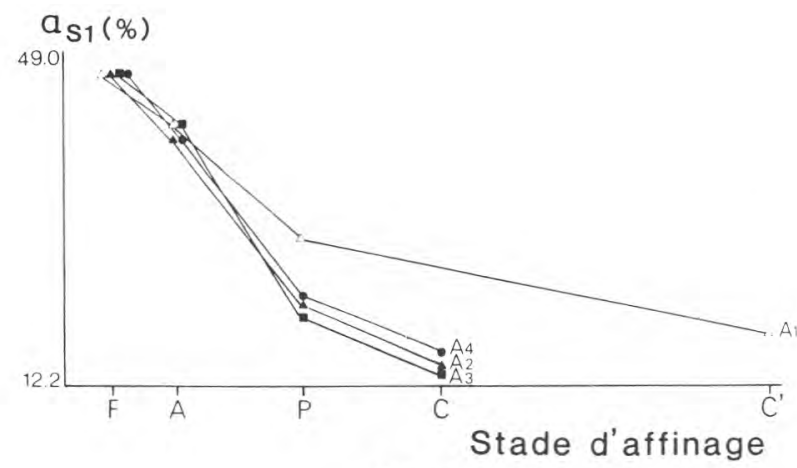

Fig. 4

Evolution du taux des caséines a S1 selon les caves d'affinage.

Evaluation of a $S 1$ casein content according to the ripening rooms.

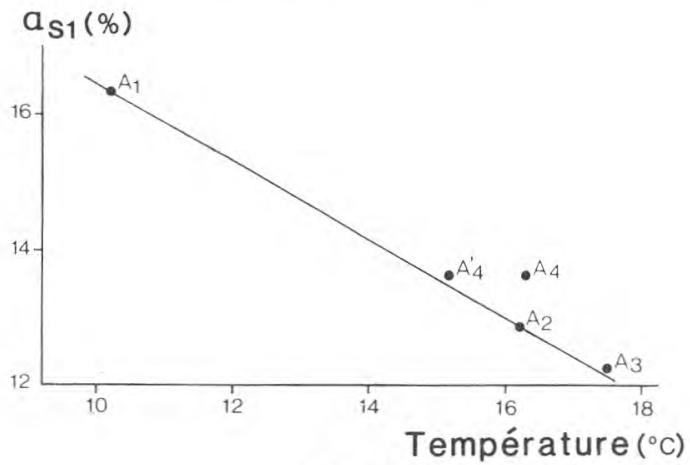

Fig. 5

Relation entre le taux moyen des caséines a S1 au stade coupe et la température moyenne des caves d'affinage.

Relationship between the average amount of a S1 caseins at stade $C$ and the average temperature of ripening rooms. 


\section{TABleau 5}

Résultats de l'analyse de variance sur les fractions azotées et les fractions caséines aux stades $P$ et $C$

Results of the analysis of variance on the nitrogen fractions and the casein fractions at ripening stages $P$ and $C$

FRACTIONS AZOTÉES

\begin{tabular}{|c|c|c|c|c|c|c|c|c|c|c|c|c|}
\hline Variables & Stades & $\begin{array}{r}\text { Fact } \\
\rightarrow\end{array}$ & $\begin{array}{l}\text { eurs } \\
\text { A }\end{array}$ & & F & & 5 & $\mathrm{AXF}$ & & XS & AXS & 3 \\
\hline \multirow[t]{2}{*}{ NS } & $\mathbf{P}$ & 1 & $\begin{array}{c}2 \\
49,5\end{array}$ & 1 & $\begin{array}{c}2 \\
26,5\end{array}$ & 1 & $\begin{array}{c}2 \\
28,5\end{array}$ & $\begin{array}{c}1 \\
\text { NS }\end{array}$ & $\begin{array}{l}1 \\
*\end{array}$ & 3,9 & $\begin{array}{c}1 \\
\text { NS }\end{array}$ & 86,4 \\
\hline & $\mathrm{C}$ & NS & & $* *$ & 26,5 & $* *$ & 24,0 & NS & ** & 7,6 & NS & 59,5 \\
\hline \multirow{2}{*}{ NPT } & $P$ & $* *$ & 26,7 & NS & & $*$ & 4,1 & NS & * & 8,0 & NS & 38,4 \\
\hline & $\mathrm{C}$ & NS & & NS & & NS & & NS & $* *$ & 19,2 & NS & 15,2 \\
\hline
\end{tabular}

FRACTIONS CASÉINES

\begin{tabular}{|c|c|c|c|c|c|c|c|c|c|c|c|c|c|}
\hline \multirow{2}{*}{ BET } & $\mathrm{P}$ & $* *$ & 35,1 & $* *$ & 10,2 & $* *$ & 15,9 & NS & NS & & NS & & 61,2 \\
\hline & $\mathrm{C}$ & NS & & $* *$ & 33,3 & NS & & NS & NS & & NS & & 33,3 \\
\hline \multirow{2}{*}{ BET D } & $\mathrm{P}$ & NS & & $*$ & 9,0 & $*$ & 5,1 & NS & NS & & NS & & 14,1 \\
\hline & C & NS & & ** & 13,2 & ** & 15,5 & NS & NS & & $* *$ & 9,8 & 8,5 \\
\hline \multirow{2}{*}{$\gamma 1$} & $\mathrm{P}$ & $* *$ & 29,7 & $* *$ & 8,3 & $* *$ & 7,1 & NS & NS & & NS & & 45,1 \\
\hline & $\mathrm{C}$ & NS & & NS & & NS & & NS & * & 7,8 & ** & 27,1 & 34,9 \\
\hline \multirow{2}{*}{$\gamma 2$} & $\mathrm{P}$ & ** & 15,1 & $* *$ & 7,5 & $* *$ & 47,1 & NS & NS & & NS & & 69,7 \\
\hline & C & NS & & $* *$ & 11,7 & $* *$ & 46,7 & NS & NS & & ** & 6,6 & 65,0 \\
\hline \multirow{2}{*}{$\gamma 3$} & $\mathrm{P}$ & ** & 25,2 & $* *$ & 6,2 & $* *$ & 32,6 & NS & NS & & NS & & 65,0 \\
\hline & C & NS & & * & 11,7 & $* *$ & 36,5 & NS & NS & & NS & & 68,2 \\
\hline \multirow{2}{*}{$\alpha \mathrm{S} 1$} & $\mathrm{P}$ & NS & & *** & 4,7 & NS & & NS & NS & & NS & & 4,7 \\
\hline & $\mathrm{C}$ & $* *$ & 32,6 & $* *$ & 10,1 & NS & & NS & NS & & NS & & 42,7 \\
\hline \multirow{2}{*}{$\alpha \mathrm{S} 1 \mathrm{I}$} & $\mathrm{P}$ & $* *$ & 35,4 & $*$ & 5,9 & NS & & NS & * & 8,5 & NS & & 52,2 \\
\hline & $\mathrm{C}$ & NS & & NS & & NS & & NS & NS & & * & 10,4 & 10,4 \\
\hline \multicolumn{14}{|c|}{$\begin{array}{ll}\text { A : Affinage } & \text { AXF : Interaction Affinage - Fromagerie } \\
\text { F : Fromagerie } & \text { FXS : Interaction Fromagerie - Saison } \\
\text { S : Saison } & \text { AXS : Interaction - Affinage - Saison } \\
\left.1: \text { Seuils de signification (NS) : non significatif : }: p<0,05 ; *^{*}: p<0,01\right) \\
2: \text { Pourcentage de variation expliqué par le facteur étudié. } \\
3: \text { Pourcentage de variation expliqué par le modèle d'analyse de variance. }\end{array}$} \\
\hline
\end{tabular}


Dans les fromages à pâte cuite où la presque totalité des enzymes coagulantes est inactivée, on peut se demander si l'hydrolyse lente des caséines $\alpha$ S1 est due à un résidu d'activité de la chymosine (GARNot et Mollé, 1987) ou à l'activité enzymatique secrétée par les microorganismes comme VISSER et de Groot-Mostert (1977) l'avaient mis en évidence sur des fabrications de fromage de Gouda. Lors de notre travail, seulement $10 \%$ des variations du taux des caséines $\alpha \mathrm{S} 1$ sont expliqués par les variations dues à la fromagerie (importance du chauffage, nature des souches microbiennes utilisées...) alors que les variations des conditions d'affinage et principalement la température des caves d'affinage semblent expliquer $32,6 \%$ des variations du taux des caséines $\alpha \mathrm{S} 1$. Ces essais ne nous permettent donc pas de mieux identifier l'origine de la protéolyse des caséines $\alpha \mathrm{S} 1$ dans ce type de fromage.

Cependant, si l'on regarde l'ensemble du phénomène, l'importance de l'hydrolyse des caséines $\alpha \mathrm{S} 1$ semble très influencée par la température de la cave d'affinage.

Le facteur affinage n'a d'influence significative sur aucune des autres caséines et fractions azotées au stade C (tabl. 5).

\section{Facteur fromagerie}

Au stade $\mathrm{C}$ de l'affinage, le facteur fromagerie s'avère agir de manière hautement significative $(\mathrm{p}<0,001)$ sur les fractions NS, $\beta, \beta$ deg., $\gamma 2, \alpha$ S1 et significative $(\mathrm{p}<0,05)$ sur la fraction $\gamma 3$. Ces résultats montrent que la protéolyse d'un fromage est grandement déterminée par la nature et la quantité des enzymes présentes dès la fromagerie, soit qu'elles proviennent du lait, soit qu'elles aient été apportées par le fromager (enzymes coagulantes, cultures microbiennes) lors de la fabrication du fromage. Chaque caillé de fromagerie possède donc un potentiel protéolytique propre.

L'influence du facteur fromagerie s'avère même être plus importante au stade $\mathrm{C}$ qu'au stade $\mathrm{P}$ pour les protéines $\beta, \beta$ deg., $\gamma 2$, $\gamma 3$ et $\alpha \mathrm{S} 1$. Ce qui tend à montrer que, lors de cette expérimentation, le déroulement de l'affinage accentue les spécificités de chaque fromagerie.

\section{Facteur saison}

Au stade $\mathrm{C}$ de l'affinage, l'influence du facteur saison apparaît hautement significative $(p<0,01)$ sur les fractions NS, $\gamma 2$ (fig. 6), $\gamma 3$ et $\beta$ deg. Ce facteur influence fortement les fractions $\gamma 2$ et $\gamma 3$. En effet, $46,7 \%$ des variations des fractions $\gamma 2$ et $56,5 \%$ des variations des fractions $\gamma 3$ sont expliquées par la saison de fabrication des fromages au stade $\mathrm{C}$ de l'affinage. On constate une très nette augmentation des proportions en caséines $y 2$ et $\gamma 3$ et $\beta$ deg. durant les mois d'août, septembre, octobre correspondant à la saison chaude de fabrication du fromage.

L'analyse en composantes principales des fractions caséine au stade coupe nous permet de comprendre précisément l'influence de la saison de fabrication des fromages sur les proportions relatives des différentes fractions caséine en fin d'affinage (fig. 7). L'évolution selon la saison (hiver : trait plein ; été : trait discontinu) des différentes proportions des fractions caséine, lorsque l'on 


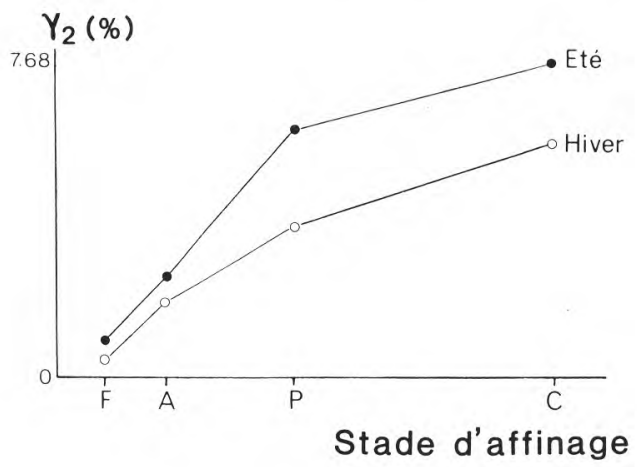

Fig. 6

Evolution du taux des caséines $\gamma 2$ en fonction du facteur saison. Evolution of the proportion of $\gamma 2$ casein according to season.

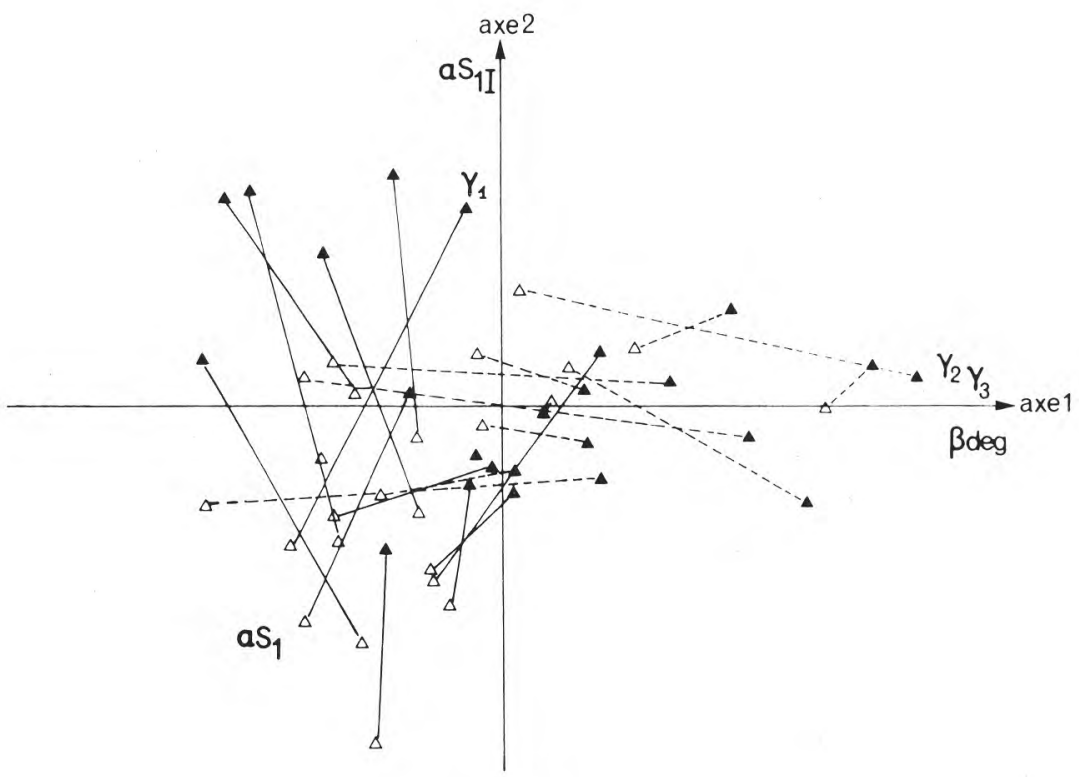

Fig. 7

Analyse en composantes principales des fractions caséines au stade coupe. Evolution en fonction de la saison (hiver - été -.---) des fractions caséines dans les fromages, lorsque l'on évolue d'un affinage à basse température $(\triangle)$ vers un affinage à $16-18^{\circ} \mathrm{C}$ $(\mathbf{\Delta})$.

Principal component analysis of casein fractions at stage C. Evolution of casein fractions in cheeses, according to the season (winter _-, summer -----) when the ripening temperature moves from low $(\triangle)$ up to a level of $16-18{ }^{\circ} \mathrm{C}(\mathbf{\Delta})$. 
évolue d'un affinage à basse température environ $10^{\circ} \mathrm{C}$ (triangle évidé) vers un affinage à $16-18^{\circ} \mathrm{C}$ (triangle plein) est très nette. Ainsi, en hiver, lorsqu'on passe d'un affinage de type basse température à un affinage à $16-18^{\circ} \mathrm{C}$, on constate que la composition moyenne des fractions caséine se déplace globalement de façon très nette selon l'axe 2, ce qui se traduit par une hydrolyse plus importante de $\alpha$ S1 et une augmentation des taux de $\alpha$ S1I et probablement de $\gamma$ 1. Par contre, avec les fromages fabriqués en été, lorsque l'on évolue d'un affinage basse température à un affinage à $16-18^{\circ} \mathrm{C}$, on observe un déplacement très net des compositions moyennes selon l'axe 1 , ce qui se traduit par une hydrolyse plus importante de la caséine $\beta$ et une augmentation des taux de $\gamma 2$ et $\gamma 3$ et $\beta$ deg.

Selon que l'on se place en hiver ou en été, l'évolution de la composition moyenne des fromages, lors du passage d'un affinage basse température à un affinage à $16-18{ }^{\circ} \mathrm{C}$, ne mène pas aux mêmes produits de dégradation.

\section{F. Influence du taux de plasmine}

Le dosage de la plasmine a été effectué sur les 24 échantillons de lait qui ont servi à fabriquer les 96 fromages. L'évolution du taux de plasmine durant les six mois de fabrication est représentée sur la figure 8 . Cette figure montre également une relation évidente entre les taux de plasmine dans les laits et les proportions de caséines $\beta$ deg., $\gamma 2$ et $\gamma 3$ dans les fromages au stade coupe et entre les taux de plasmine et les taux d'azote soluble dans les fromages à ce même stade.

L'hydrolyse de la caséine $\beta$ par la plasmine donne naissance d'une part aux caséines $\gamma$ visibles en électrophorèse et d'autre part aux protéoses-peptones présentes dans la partie azote soluble du fromage. Nous avons ainsi établi une corrélation hautement significative $(r=0,661, p<0,001)$ entre les taux d'azote soluble dans les fromages en fin d'affinage et la quantité de plasmine mesurée dans les laits de fabrication.

Le taux de plasmine est plus élevé pendant les mois d'été (août, septembre, octobre) que pendant les premiers mois de l'année (février, mars, avril).

Les variations saisonnières du taux de plasmine confirment les remarques faites par plusieurs auteurs (DAVIES et LAW, 1977 ; BARRY et DONNELLY, 1980) sur les évolutions du taux des caséines $\gamma$ en fonction du stade de lactation. La mesure du taux de plasmine en fonction de la saison a aussi été étudiée par Richardson (1983) et Korycka-Dahl et al. (1983). ShaAr (1985) a même montré que le stade de lactation est le facteur qui influe le plus sur l'activité de la plasmine. Plus récemment Benslimane (1986) a pu différencier l'effet dû au stade de lactation et l'effet dû à la saison, sur l'activité de la plasmine, malgré la superposition assez large de ces deux effets.

L'évolution saisonnière du taux de plasmine dans les laits semble donc expliquer pour une grande partie l'évolution saisonnière de l'hydrolyse de la caséine $\beta$ pour donner les caséines $\gamma 2$ et $\gamma 3$ et, dans une moindre mesure, la caséine appelée $\beta$ deg. Avec cette forte influence du taux de plasmine sur la composition des fractions caséines en fin d'affinage, il apparaît intéressant de savoir si cette influence est décelable précocement dans les fromages. Dans ce 

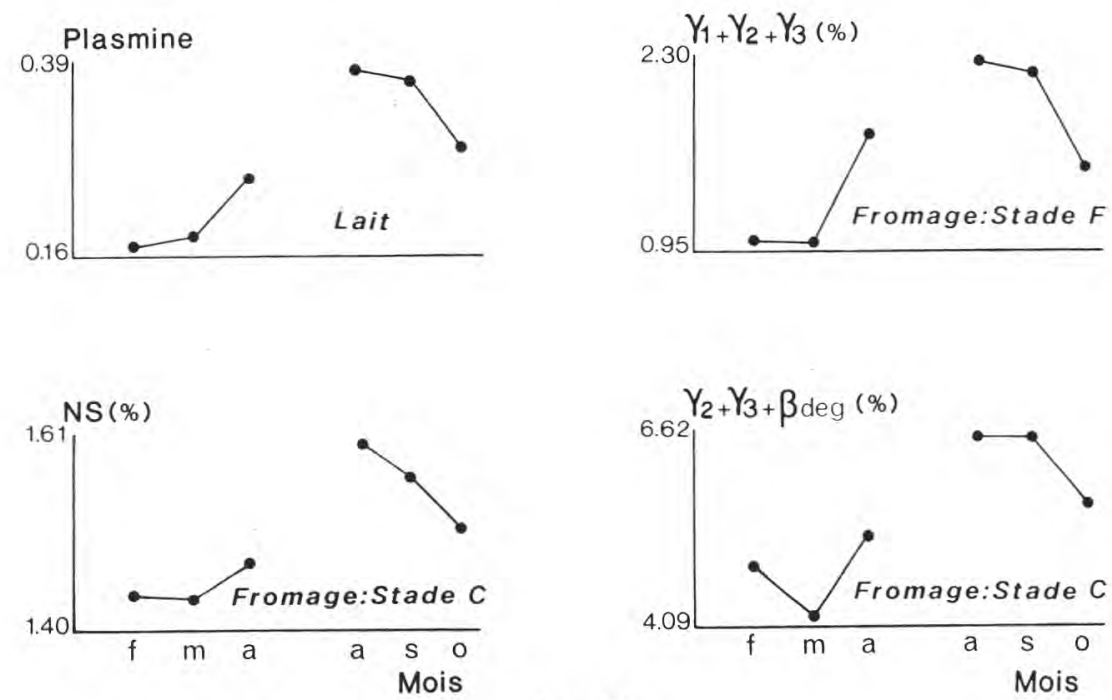

Fig. 8

Evolution comparée du taux de plasmine dans les laits et des proportions de certains produits de dégradation de la caséine $\beta$ dans les fromages aux stades $F$ et $C$.

Comparative evolution of milk plasmin content in milk and proportions of some $\beta$-casein degradation products in cheeses at stages $F$ and $C$.

but, nous avons représenté (fig. 8) l'évolution mensuelle de la somme des caséines $\gamma 1, \gamma 2$ et $\gamma 3$ présentes dans les fromages 20 heures après leur fabrication. L'évolution des caséines $\gamma$ apparaît suivre nettement celle du taux de plasmine dans le lait. L'influence du taux de plasmine sur l'hydrolyse de la caséine $\beta$ semble donc étre très précoce.

Les proportions de caséine $\gamma$ mesurées 24 heures après la fabrication sont sans doute grandement influencées par la proportion de ces caséines présentes dans le lait et produites durant sa maturation. L'importance de cette maturation qui reste encore obscure doit donc varier avec la saison de fabrication et le stade de lactation des troupeaux (Benslimane, 1986). Une meilleure connaissance du potentiel enzymatique des laits devrait permettre de mieux appréhender le problème de leur maturation et de mieux prévoir l'importance de certaines protéolyses finales dans le fromage.

\section{Conclusion}

Cette étude a permis de déterminer la nature, la vitesse et l'importance de la protéolyse des caséines pendant l'affinage du Comté.

Elle a montré aussi le rôle prépondérant de la plasmine dans l'hydrolyse de la caséine $\beta$ et explique en grande partie les variations saisonnières des taux 
des produits de dégradations de cette caséine $(\gamma 2, \gamma 3$ et $\beta$ deg.), alors que l'hydrolyse de la caséine $\alpha$ S1 semble plutôt être influencée par les conditions d'affinage du fromage et plus particulièrement par les variations de température.

L'étude des fromages en fín d'affinage montre que, pour toutes les protéines étudiées, à l'exception de la caséine $\alpha \mathrm{S} 1$, l'incidence de la fromagerie (composition du lait, nature des ferments lactiques, technologie de fabrication) est plus importante au niveau de la protéolyse que les conditions d'affinage.

La caséine $\gamma 1$ apparaît dans cette étude sans lien net avec les caséines $\gamma 2$ et $\gamma 3$ et semble posséder un comportement différent des autres produits de dégradation de la caséine $\beta$. Déjà Kaminogawa et al. (1972), dans une étude sur la plasmine, avaient observé un comportement différent des $\gamma 1$ par rapport aux $\gamma 2$ et $\gamma 3$.

Les variations cycliques du taux de plasmine pourraient expliquer en partie l'apparition de certains défauts du Gruyère de Comté comme la lainure, qui apparaissent eux aussi de façon cyclique pendant les mois d'été où le taux de plasmine est le plus élevé. Ce travail a ainsi permis de prouver clairement qu'il existe un lien saisonnier entre les variations du taux d'une enzyme, la plasmine du lait, et l'état de protéolyse des caséines en fin d'affinage.

D'une manière générale, il semble que la plasmine ait une place particulièrement importante dans la protéolyse des fromages à pâte cuite pressée. En effet, cette enzyme très thermostable verrait même son activité croître après traitement thermique, soit par inactivation des inhibiteurs naturels (NomEN, 1975) ou bien par suite d'une activation plasminogène plasmine consécutive au chauffage (Rollema et al., 1981). Il semble donc que la recherche d'une normalisation du taux de plasmine dans les laits destinés à la fabrication du Gruyère pourrait prévenir une partie des défauts de ces fromages.

Reçu le 23 mai 1986

Accepté pour publication le 7 novembre 1986

\section{Remerciements}

Nous tenons à exprimer nos remerciements à M. RIBADEAU DUMAS, Directeur du Laboratoire de Biochimie et Technologie laitières de Jouy-en-Josas, pour nous avoir accueilli dans son laboratoire afin d'acquérir la maîtrise de la méthode électrophorétique, ainsi que pour la correction du manuscrit.

\section{Références bibliographiques}

Bachacou J., Masson J.P., Millier C., 1981. Manuel de la programmation statistique Amance 81. Ed. Dép. Biométrie CNRF-INRA, Champenoux.

Barry J.G., Donnelly W.J., 1980. Casein compositional studies 1. The composition of casein from Friesian herd milks. J. Dairy Res., 47, 71-82. 
Benslimane S., 1986. Variations de l'activité de la plasmine et du plasminogène dans les laits de vaches montbéliarde et dans les fromages de type Comté. Thèse de doctorat $3^{\mathrm{e}}$ cycle, Université de Besançon.

Berdague J.L., 1986. Affinage du Gruyère de Comté. Thèse de doctorat de l'Université de Bourgogne.

Creamer L.K., Richardson B.C., 1974. Identification of the primary degradation product of $\alpha$-S1 casein in Cheddar cheese. N.Z.J. Dairy Sci. Technol., 9, 9-13.

Creamer L.K., 1974. Casein degradation in Gouda and Cheddar cheese. J. Dairy Sci., 58, $287-$ 292.

Davies D.T., LAw A.J.R., 1977. The composition of whole casein from the milk of Ayrshire cows. J. Dairy Res., 44, 447-454.

Fox P.F., Guiney J., 1973. Casein micelle structure : susceptibility of various casein systems to proteolysis. J. Dairy Res., 40, 229-234.

Garnot P., Molle D., 1987. Heat-stability of milk-clotting enzymes in conditions encountered in Swiss cheese making. J. Food Sci., 52 (1).

Grappin R., Rank T.C., Ol.son N.F., 1985. Primary proteolysis of cheese proteins during ripening. A review. J. Dairy Sci., 68, 531-540.

Grappin R., Berdague J.L., Dasen A., Jeunet R., 1987. Affinage et qualité du Gruyère de Comté. I : Protocole expérimental. Caractérisation des types d'affinage et des ateliers de fabrication. Lait, 67 (2), 219-235.

Gripon J.C., Desmazeaud M.J., Le Bars D., Bergère J.L., 1975. Etude du rôle des microorganismes et des enzymes au cours de la maturation des fromages. II. Influence de la présure commerciale. Lait, 55, 502-516.

Kaminogawa S., Yamauchi K., 1972. Decomposition of $\beta$-casein by milk protease. Similarity of the decomposed products to temperature sensitive and R-caseins. Agric. Biol. Chem., 36, 255260.

Korycka-Dahl M., Ribadeau Dumas B., Chene N., Martal J., 1983. Plasmin activity in milk. $I$. Dairy Sci., 66, 704-711.

Kuchroo C.N., Fox P.F., 1982b. Fractionation of the water soluble nitrogen from Cheddar cheese : chemical methods. Milchwissenschaft, 37, 651-653.

Microstat ITCF, 1984. Service des études statistiques et informatiques. Station Expérimentale ITCF, Boigneville, 91720 Maisse.

Mulvihill D.M., Fox P.F., 1980. Proteolysis of $\alpha$ S1-casein by chymosin in dilute $\mathrm{NaCl}$ solutions and in Cheddar cheese. Ir. J. Food Sci. Technol., 4, 13-23.

Noomen A., 1975. Proteolytic activity of milk protease in raw and pasteurized cow's mikl. Neth. Milk Dairy J., 29, 153-161.

Richardson B.C., Pearce K.H., 1981. The determination of plasmin in dairy products. N.Z.J. Dairy Sci. Technol., 16, 209-220.

RichaRdSON B.C., 1983. Variation of the concentration of plasmin and plasminogen in bovine milk with lactation. N.Z.J. Dairy Sci. Technol., 18, 247-252.

Rollema H.S., VisSer S., Poll J.C., 1981. On the determination purification and characterization of the alkaline proteinase from bovine milk. Neth. Milk Dairy J., 35, 396-399.

SchaAR J., 1985. Plasmin activity and proteose-peptone content of individual milks. J. Dairy Res., $52,369-378$.

Trieu-Cuot P., Gripon J.C., 1983. Etude électrophorétique de la protéolyse au cours de l'affinage des fromages à pâte persillée du type bleu d'Auvergne. Lait, 63, 116-128.

Uriel J., 1966. Méthode d'électrophorèse dans des gels d'acrylamide-agarose. Bull. Soc. Chim. Biol., 48, 969-982.

Visser F.M.W., DE Groot-Mostert A.E.A., 1977. Contribution of enzymes from rennet, starter bacteria and milk to proteolysis and flavour development in Gouda cheese. 4. Protein breakdown : a gel electrophoretical study. Neth. Milk Dairy J., 31, 247-261. 